\title{
BMJ Open BOugie or stylet in patients UnderGoing Intubation Emergently (BOUGIE): protocol and statistical analysis plan for a randomised clinical trial
}

\author{
Brian Driver (1) , ${ }^{1}$ Matthew W Semler (1) , ${ }^{2}$ Wesley H Self, ${ }^{3}$ Adit A Ginde, ${ }^{4}$ \\ Sheetal Gandotra (1) , 5 Stacy A Trent, ${ }^{4,7}$ Lane M Smith, ${ }^{8}$ John P Gaillard, ${ }^{8}$ \\ David B Page ${ }^{9,10}$ Micah R Whitson, ${ }^{9,10}$ Derek J Vonderhaar, ${ }^{11}$ AM Joffe, ${ }^{12}$ \\ Jason R West (1) , ${ }^{13}$ Christopher Hughes, ${ }^{14}$ Janna S Landsperger, ${ }^{2}$ \\ Michelle P Howell, ${ }^{4}$ Derek W Russell (D) , ${ }^{5,6}$ Swati Gulati, ${ }^{5,6}$ Itay Bentov, ${ }^{12}$ \\ Steven Mitchell, ${ }^{15}$ Andrew Latimer, ${ }^{15}$ Kevin Doerschug, ${ }^{16}$ Vikas Koppurapu, ${ }^{16}$ \\ Kevin W Gibbs, ${ }^{17}$ Li Wang, ${ }^{18}$ Christopher John Lindsell, ${ }^{18}$ David Janz, ${ }^{19}$ \\ Todd W Rice, ${ }^{2}$ Matthew E Prekker, ${ }^{1,20}$ Jonathan D Casey, ${ }^{2}$ BOUGIE Investigators\# \\ and the Pragmatic Critical Care Research Group
}

To cite: Driver B, Semler MW, Self WH, et al. BOugie or stylet in patients UnderGoing Intubation Emergently (BOUGIE): protocol and statistical analysis plan for a randomised clinical trial. BMJ Open 2021;11:e047790. doi:10.1136/ bmjopen-2020-047790

- Prepublication history and additional supplemental material for this paper are available online. To view these files, please visit the journal online (http://dx.doi.org/10.1136/ bmjopen-2020-047790).

$\mathrm{BD}$ and MWS contributed equally.

MEP and JDC contributed equally.

Received 08 December 2020 Accepted 09 May 2021

Check for updates

(C) Author(s) (or their employer(s)) 2021. Re-use permitted under CC BY-NC. No commercial re-use. See rights and permissions. Published by BMJ.

For numbered affiliations see end of article.

Correspondence to

Dr Brian Driver;

briandriver@gmail.com

\section{ABSTRACT}

Introduction Intubation-related complications are less frequent when intubation is successful on the first attempt. The rate of first attempt success in the emergency department (ED) and intensive care unit (ICU) is typically less than $90 \%$. The bougie, a semirigid introducer that can be placed into the trachea to facilitate a Seldinger-like technique of tracheal intubation and is typically reserved for difficult or failed intubations, might improve first attempt success. Evidence supporting its use, however, is from a single academic ED with frequent bougie use. Validation of these findings is needed before widespread implementation. Methods and analysis The BOugie or stylet in patients Undergoing Intubation Emergently trial is a prospective, multicentre, non-blinded randomised trial being conducted in six EDs and six ICUs in the USA. The trial plans to enrol 1106 critically ill adults undergoing orotracheal intubation. Eligible patients are randomised 1:1 for the use of a bougie or use of an endotracheal tube with stylet for the first intubation attempt. The primary outcome is successful intubation on the first attempt. The secondary outcome is severe hypoxaemia, defined as an oxygen saturation less than $80 \%$ between induction until 2 min after completion of intubation. Enrolment began on 29 April 2019 and is expected to be completed in 2021.

Ethics and dissemination The trial protocol was approved with waiver of informed consent by the Central Institutional Review Board at Vanderbilt University Medical Center or the local institutional review board at an enrolling site. The results will be submitted for publication in a peerreviewed journal and presented at scientific conferences. Trial registration number ClinicalTrials.gov Registry (NCT03928925).

\section{INTRODUCTION}

Tracheal intubation of critically ill adults is frequently performed in the emergency

\section{Strengths and limitations of this study}

This protocol provides a detailed description of the largest pragmatic trial of bougie use in emergency airway management to be conducted to date.

- Broad eligibility criteria, diverse prior experience with a bougie among operators and conduct in the emergency department and intensive care unit at multiple centres will increase the external validity of the findings.

- Patients, clinicians and investigators are not blinded to study group assignment after randomisation.

department (ED) and intensive care unit (ICU). Successful intubation on the first attempt has been associated with a lower incidence of peri-intubation complications. ${ }^{1-4}$ However, less than $90 \%$ of patients are intubated on the first attempt in most settings outside of the operating room, highlighting an opportunity for improvement. ${ }^{5-7}$

Emergency tracheal intubation is commonly performed in three discrete steps. First, medications are administered to facilitate optimal intubating conditions (induction). Second, a laryngoscope is inserted into the patient's mouth and a direct or indirect video view of glottic structures is obtained (laryngoscopy). Third, an endotracheal tube is placed in the mouth and advanced past the vocal cords into the trachea (intubation). Two commonly used devices that aid in placing the endotracheal tube include: a stylet (a malleable, metal rod preloaded inside the endotracheal tube 
to facilitate navigation of the upper airway) or a bougie (a thin, plastic introducer passed into the trachea which serves as a guide for passage of the endotracheal tube). When using a stylet, the endotracheal tube and stylet are passed into the trachea together. When using a bougie, the bougie is first passed into the trachea and then the endotracheal tube is advanced over the bougie using a Seldinger-like technique. There is substantial variation between clinicians as to whether they select the stylet or the bougie for the first intubation attempt. ${ }^{58}$ For some physicians, the bougie is used primarily as a rescue device in the event difficulty is encountered in laryngoscopy or passage of the endotracheal tube with stylet. Other physicians use a bougie routinely on the first attempt at tracheal intubation. $^{89}$

To our knowledge, only one prior randomised trial has compared rates of successful intubation on the first attempt outside of the operating room with use of a bougie versus use of endotracheal tube with stylet: the single-centre Bougie Use in Emergency Airway Management (BEAM) trial. That study showed a higher rate of successful intubation on the first attempt with use of a bougie $(98 \%)$ compared with use of an endotracheal tube with stylet $(87 \%)$ in adult ED patients (absolute difference $11 \%, 95 \%$ CI $7 \%$ to $14 \%) .{ }^{10}$ However, it is possible that these findings reflect increased institution-specific comfort with bougie use compared with the endotracheal tube and stylet-operators reported using a bougie in approximately $80 \%$ of intubations before the trial. ${ }^{8}$ It is unknown if the results of the BEAM trial will generalise to other settings where operators have less experience using the bougie and have greater experience using an endotracheal tube with stylet during the first attempt at intubation.

\section{METHODS AND ANALYSIS}

This manuscript was written in accordance with Standard Protocol Items: Recommendations for Interventional Trials (SPIRIT) guidelines (see table 1 and online supplemental file 1 , section 1$).{ }^{11}$

\section{Patient and public involvement}

We did not involve patients or the public in the design of the study.

\section{Study design}

The BOugie or stylet in patients UnderGoing Intubation Emergently (BOUGIE) trial is a pragmatic, multicenter, unblinded, parallel-group, randomised trial comparing use of a bougie to use of an endotracheal tube with stylet for the first attempt at tracheal intubation among critically ill adults in the ED and ICU. The primary outcome is successful intubation on the first attempt. An independent data and safety monitoring board (DSMB) is monitoring the progress and safety of the trial. Study sites and investigators are listed in online supplemental file 1, sections 2 and 3 .

\section{Study population}

The inclusion criteria for the trial are:

1. Patient is located in a participating unit of an adult hospital.

2. Planned procedure is tracheal intubation with sedative administration (or tracheal intubation without sedative administration in patients with decreased level of consciousness, cardiac arrest or respiratory arrest).

3. Planned operator is a clinician expected to routinely perform tracheal intubation in the participating unit.

4. Planned laryngoscopy device is a non-hyperangulated laryngoscope blade.

The exclusion criteria for the trial are:

1. Patient is pregnant.

2. Patient is a prisoner.

3. Urgency of intubation precludes safe performance of study procedures.

4. Operator feels an approach to intubation other than use of a bougie or use of an endotracheal tube with stylet would be best for the care of the patient.

5. Operator feels use of a bougie is required or contraindicated for the care of the patient.

6. Operator feels use of an endotracheal tube with stylet is required or contraindicated for the care of the patient.

The original inclusion criteria specified that patients must be at least 18 years old to be eligible. With approval from the Central Institutional Review Board at Vanderbilt University Medical Center, trial inclusion and exclusion criteria were amended on 16 January 2020 to allow the enrolment of patients less than 18 years of age. Because the identity and age of critically ill patients presenting to the ED are sometimes unknown (eg, a patient with cardiac arrest presenting by ambulance without family), this criterion was revised to include patients located in a participating unit of an adult hospital. We anticipate that a small number of patients whose identity and age are unknown, who are judged by treating clinicians to be an adult and enrolled in the trial, will later be determined to be less than 18 years old.

\section{Randomisation and treatment allocation}

Patients are randomised in a 1:1 ratio to undergo intubation using a bougie or using an endotracheal tube with stylet for the first attempt in permuted blocks of two, four or six, stratified by study site. Study-group assignments are generated using a computerised randomisation sequence, placed in sequentially numbered opaque envelopes and distributed to enrolling sites. Before opening the envelope, the operator determines that the patient meets all inclusion criteria and no exclusion criteria. The operator documents whether they plan to use a video laryngoscope or a direct laryngoscope by checking a box on the front of the envelope. The operator then opens the envelope. Patients are considered to be enrolled once the operator opens the envelope to reveal study group assignment. Thus, group assignment is concealed until after documentation of laryngoscope choice and patient 


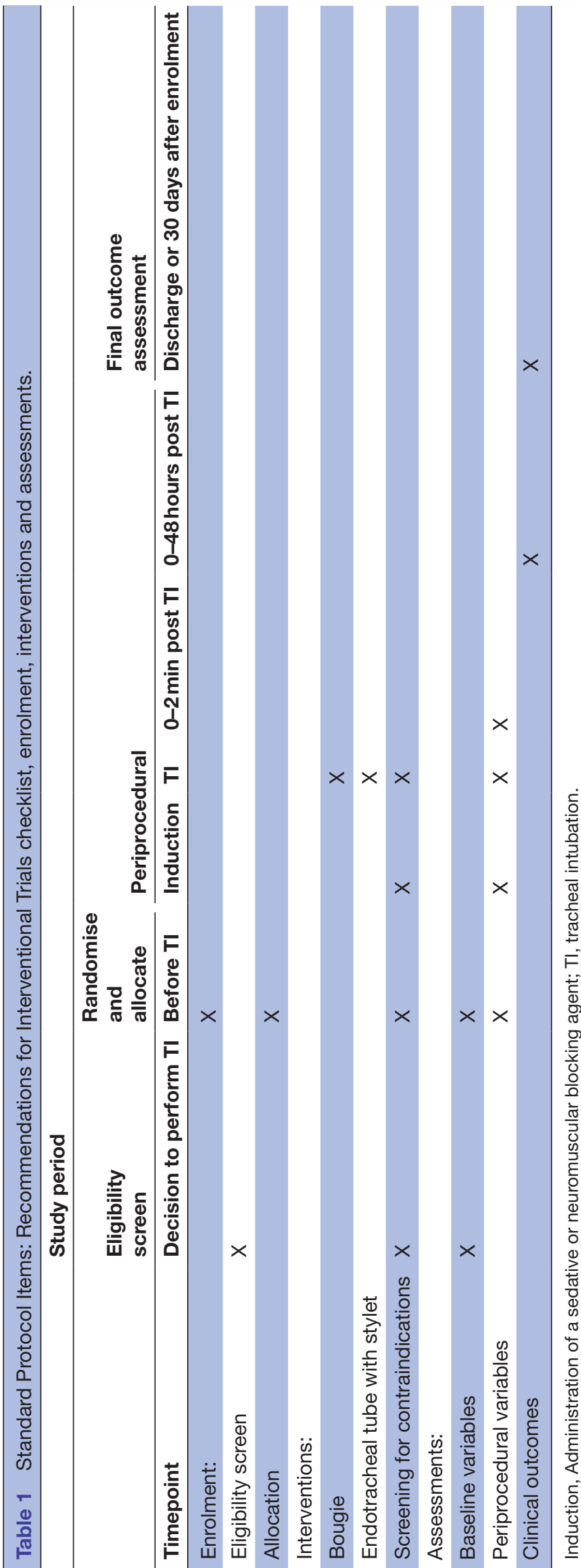

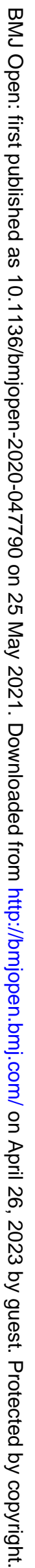


enrolment. Patients who are screened and excluded will be reported with trial results using a Consolidated Standards of Reporting Trials diagram. After enrolment and randomisation, patients, treating clinicians and study personnel are not blinded to study group assignment.

\section{Study interventions}

Training

Before beginning enrolment at a site, operators at each site received a $30 \mathrm{~min}$ in-person lecture and watched a 6 min training video which demonstrated best practices for intubation with both a bougie and endotracheal tube with stylet. These materials are available from the authors on request.

\section{Bougie group}

For patients assigned to the bougie group, operators are instructed to use a bougie on the first attempt at laryngoscopy and tracheal intubation. If the bougie is successfully placed in the trachea, an assistant is instructed to load the endotracheal tube (without a stylet) over the bougie. The operator is instructed to, without removing the laryngoscope from the mouth, advance the tube through the vocal cords to the desired depth in the trachea. If resistance is encountered when passing the endotracheal tube over the bougie, the tube is be retracted $2 \mathrm{~cm}$, rotated $90^{\circ}$ counterclockwise to orient the bevel tip of the tube vertically and readvanced into the trachea. With the operator or an assistant manually stabilising the endotracheal tube, the bougie is withdrawn from the endotracheal tube before ventilation. Confirmation of correct endotracheal tube placement is deferred to clinicians; detection of end-tidal carbon dioxide is the standard of care at participating institutions.

This trial evaluates the use of a straight, semirigid bougie. Experts report that less-rigid bougies packaged in a curled position are more difficult to advance through the glottic opening. ${ }^{12}$ Participating units use a straight bougie at least $60 \mathrm{~cm}$ in length; a coudé tip is favoured but not required. Operators may choose whether and how to bend the bougie prior to intubation.

\section{Endotracheal tube with stylet group}

For patients assigned to the endotracheal tube with stylet group, operators are instructed to use an endotracheal tube with stylet on the first attempt at laryngoscopy and tracheal intubation. The shape and curvature of the endotracheal tube with stylet is determined by the operator, however a 'straight-to-cuff' shape and a distal bend angle of $25^{\circ}-35^{\circ}$ is encouraged. If there is difficulty passing the endotracheal tube, the operator is instructed to manipulate the tube as needed, including slight retraction and rotation. The stylet remains within the endotracheal tube until the tube is within the trachea. Confirmation of correct endotracheal tube placement is deferred to clinicians; detection of end-tidal carbon dioxide is the standard of care at participating institutions.
Subsequent attempts at laryngoscopy and intubation and cointerventions

Study group assignment determines only the device to be used on the first attempt at laryngoscopy and tracheal intubation. All other aspects of the intubation procedure are at the discretion of treating clinicians, including choice of endotracheal tube diameter, patient position, approach to preoxygenation, approach to ventilation and oxygenation between induction and intubation and devices used after the first intubation attempt. For laryngoscopes capable of both video-assisted and direct laryngoscopy, the use of the video screen during intubation is at the discretion of the operator. After the first attempt at laryngoscopy and tracheal intubation, the operator may use any other method of intubation, including use of an endotracheal tube with stylet in the bougie group or use of a bougie in the endotracheal tube with stylet group. In either group, treating clinicians may, at any point, use any device they feel is required to ensure optimal care of the patient regardless of study group assignment. The approach to the initial attempt at laryngoscopy and intubation and any cointerventions are prospectively collected and will be reported.

Coenrolment in other randomised trials is permitted as the use of randomisation facilitates balance between study arms, reduces the likelihood of any systematic effects on intubation success rates and allows for evaluation of the main effects in this trial.

\section{Data collection}

An observer, not directly involved with the intubation procedure, collects data for key periprocedural outcomes, including successful intubation on the first attempt, time between induction and successful intubation, arterial oxygen saturation and systolic blood pressure at induction and the lowest values for arterial oxygen saturation and systolic blood pressure between induction and 2 min following intubation. The background of trained observers depends on local context and may include either clinical professionals (eg, physicians or nurses) or research study personnel. All observers received training on study procedures and data element definitions.

Immediately after the procedure, operators complete a paper data collection form to document the approach to oxygen administration and use of ventilation for preoxygenation and between induction and laryngoscopy, laryngoscope used, Cormack-Lehane grade of glottic view ${ }^{13}$, laryngoscope video screen use (if applicable), reason for the failure to intubate on the first attempt (if applicable), subsequent intubation methods, difficult airway characteristics (cervical collar, glottic view obscured by body fluids, facial trauma) and complications of intubation (cardiac arrest, heart rate $<40$ beats per minute, oesophageal intubation, airway trauma, witnessed aspiration). Operators record their specialty and training level and self-report the number of prior intubations, overall and with a bougie, at the time of each study intubation. 
Study personnel review the medical record to collect data on baseline characteristics, pre and post laryngoscopy management and clinical outcomes. The following variables are collected:

1. Baseline: Age, gender, height, weight, race, ethnicity, APACHE (acute physiology and chronic health evaluation) II Score, most recent preprocedural Glasgow Coma Score, active medical problems at the time of intubation, active and chronic comorbidities complicating intubation, whether the primary diagnosis was trauma related, indication for intubation, non-invasive positive pressure ventilation and high flow nasal cannula use, vasopressor use in the hour preceding enrolment, presence of sepsis (defined as life-threatening organ dysfunction caused by a dysregulated host response to infection) or septic shock (defined as presence of sepsis plus vasopressor requirement to maintain a mean arterial pressure of $65 \mathrm{~mm} \mathrm{Hg}$ or greater and serum lactate $>2 \mathrm{mmol} / \mathrm{L}$ in the absence of hypovolemia) at the time of enrolment, the highest fraction of inspired oxygen delivered $\left(\mathrm{FiO}_{2}\right)$ in the hour preceding enrolment and whether or not this was a reintubation (defined as a patient who had been extubated from invasive mechanical ventilation within the prior 72 hours).

2. Periprocedural: Type and dose of neuromuscular blocker; laryngoscope device used, blade shape and size for first attempt; total number of intubation attempts and presence of any of the following difficult airway characteristics: vomiting, witnessed aspiration, upper gastrointestinal haemorrhage, epistaxis or oral bleeding, upper airway mass, infection or trauma, head and neck radiation, obesity (body mass index $>30 \mathrm{~kg}$ / $\mathrm{m}^{2}$ ), limited neck mobility, limited mouth opening, history of obstructive sleep apnea or other.

3. 0-48 hours: Cardiac arrest within 1 hour of intubation, presence or absence of pneumothorax on first chest film obtained within 48 hours after intubation and systolic blood pressure, oxygen saturation, $\mathrm{FiO}_{2}$ and positive end expiratory pressure delivered at 24 hours after enrolment.

4. In-hospital outcomes: Ventilator-free days, ICU-free days and 28 days in-hospital mortality.

\section{Primary outcome}

The primary outcome is successful intubation on the first attempt. Successful intubation on the first attempt is defined as placement of an endotracheal tube in the trachea following: (1) a single insertion of a laryngoscope blade into the mouth and (2) either a single insertion of a bougie into the mouth followed by a single insertion of an endotracheal tube into the mouth or a single insertion of an endotracheal tube with stylet into the mouth.

The primary outcome is collected by a trained observer using a structured data collection form that records the number of insertions of the laryngoscope blade, bougie and endotracheal tube into the patient's mouth. If data from the independent observer about the primary outcome are missing, the operator's self-report of successful intubation on the first attempt will be used. If documentation of successful intubation on the first attempt is discordant between the independent observer and the operator, data from the independent observer will take precedence.

\section{Secondary outcome}

The secondary outcome is the incidence of severe hypoxaemia, defined as an oxygen saturation less than $80 \%$ during the time interval from induction to $2 \mathrm{~min}$ after completion of tracheal intubation.

\section{Exploratory outcomes}

- Cormack-Lehane grade of glottic view.

- Number of laryngoscopy attempts.

- Number of attempts at passing the bougie.

- Number of attempts at passing the endotracheal tube.

- Duration of intubation: The start of the procedure will be defined as either the time of first sedative administration or, among patients who do not receive a sedative, the time of initiation of laryngoscopy. The end of the procedure will be defined as the time of the final placement of an endotracheal tube within the trachea.

- Whether the video laryngoscope screen was viewed, among intubations where the operator used a video laryngoscope.

- Incidence of mechanical intubation complications, including:

Oesophageal intubation.

Operator-reported aspiration during the procedure.

Airway trauma (injury to oropharyngeal, glottic or thoracic airway structures).

- Cardiac arrest within 1 hour following intubation.

- Incidence of peri-intubation cardiovascular collapse, defined as one or more of:

New systolic blood pressure $<65 \mathrm{~mm} \mathrm{Hg}$ between induction and $2 \mathrm{~min}$ following intubation.

New or increased vasopressor between induction and 2 min following intubation.

Cardiac arrest within 1 hour of intubation.

Death within 1 hour of intubation.

- ICU-free days in the first 28 days (see online supplemental file 1 , section 4 ).

- Ventilator-free days in the first 28 days (see online supplemental file 1 , section 5 ).

- All-cause, in-hospital mortality at 28 days.

\section{Sample size estimation}

There is no established minimum clinically important difference in successful intubation on the first attempt. A prior single-centre randomised trial reported an absolute difference of $11 \%$ in successful intubation on the first attempt between the bougie and endotracheal tube with stylet groups. Because this trial was performed in an ED where the majority of first intubation attempts used a bougie, we anticipated a potentially smaller difference 
between groups in this multicenter trial conducted in a broader range of clinical settings with a broader range of operators. Therefore, the current trial was designed to detect a $6 \%$ absolute difference between groups in the proportion of patients who experience successful intubation on the first attempt. For two inexpensive interventions already routinely available and used in practice, the minimally clinically significant difference that would be expected to change practice is unknown. However, an absolute difference of $6 \%$ in successful intubation on the first attempt is similar to or smaller than the difference considered to be clinically meaningful in the design of prior airway management trials. ${ }^{7} 1014$ Assuming $84 \%$ of patients in the endotracheal tube with stylet group experience successful intubation on the first laryngoscopy attempt, detecting a $6 \%$ absolute increase in successful intubation on the first attempt with $80 \%$ power at a twosided alpha level of 0.05 would require enrolment of 1050 patients (525 per group). Anticipating missing data for $5 \%$ of patients or less, we will plan to enrol a total of 1106 patients (553 per group).

\section{DSMB and interim analysis}

A DSMB composed of four clinical trials experts with backgrounds in critical care medicine, anaesthesia and emergency medicine has overseen the design of the trial and is monitoring its conduct. The DSMB reviewed a single interim analysis, prepared by the study biostatistician, on 4 February 2020, at the anticipated halfway point of the trial after enrolment of 553 patients, and recommended continuing the trial to completion without alteration. The stopping boundary for efficacy was prespecified as a $p$ value of 0.001 or less for the difference in the incidence of the primary outcome between groups tested, using a $\chi^{2}$ test. This conservative Haybittle-Peto boundary was selected to allow the final analysis to be performed using an unchanged level of significance $(\mathrm{p}<0.05)$. The recommended stopping boundary for safety was a $\mathrm{p}<0.025$ comparing the incidence of oesophageal intubation and separately the incidence of airway trauma between groups, using a $\chi^{2}$ test. The DSMB retains the authority to stop the trial at any point, request additional data or interim analyses or request modifications of the study protocol to protect patient safety. The DSMB charter is available in online supplemental file 1, section 6. Patient privacy and data storage details are listed in online supplemental file 1 , section 7 .

\section{Statistical analysis principles}

Analyses will be conducted following reproducible research principles using R ( $\mathrm{R}$ Foundation for Statistical Computing, Vienna, Austria). ${ }^{15}$ Continuous variables will be reported as mean $\pm \mathrm{SD}$ deviation or median and IQR; categorical variables will be reported as frequencies and proportions. Between-group comparisons will be made with the Wilcoxon rank sum test for continuous variables and the $\chi^{2}$ test for categorical variables. We will also present absolute between-group differences with associated $95 \%$ CIs. A two-sided $p$ value of $<0.05$ will be used to indicate statistical significance; with just one primary outcome, no adjustment for multiplicity will be made. For secondary and exploratory analyses, emphasis will be placed on the magnitude of differences between groups rather than statistical significance.

\section{Main analysis of the primary outcome}

The main analysis will be an unadjusted, intention-to-treat comparison of successful intubation on the first attempt between patients randomised to the bougie group and patients randomised to the endotracheal tube with stylet group, using a $\chi^{2}$ test.

\section{Secondary analyses of the primary outcome}

\section{Multivariable modeling to account for covariates}

To account for relevant covariates, we will develop a generalised linear mixed effects model using a logit link function with the primary outcome as the dependent variable, study site and operator as random effects and fixed effects of study group and the following prespecified baseline covariates: age, sex, race, body mass index, operator experience quantified as the operator's total number of prior intubations and location of intubation (ED vs ICU). We will then construct a model with the following additional factors that may be interpreted as baseline covariates but which are unable to be assessed until after randomisation: use of a video versus direct laryngoscope; presence of $\geq 1$ difficult airway characteristic (obesity, body fluids obscuring glottic view, cervical immobilisation or facial trauma) and Cormack-Lehane grade 1 vs grade 2, 3 or 4 laryngeal view. All continuous variables will be modelled assuming a non-linear relationship to the outcome using restricted cubic splines with between 3 and 5 knots.

\section{Effect modification}

We will examine whether prespecified variables modify the effect of bougie versus endotracheal tube with stylet use on the primary outcome using a formal test of interaction between group assignment and effect modifier in the above models. Because this study is not formally designed or powered to test for interaction, a less conservative $p$ value for the interaction term will be used, with values less than 0.10 considered suggestive of a potential interaction and values less than 0.05 considered to confirm an interaction. We will examine whether the following baseline variables modify the effect of study group on the primary outcome:

1. Operator experience at the time of each enrolment.

a. Total number of previous intubations performed by operator.

b. Number of previous intubations performed by operator using a bougie.

c. Proportion of previous intubations performed by the operator that were performed using a bougie.

2. Location (ED vs ICU).

3. Indication for intubation (trauma vs medical). 
4. Difficult airway, defined as one or more of the following difficult airway characteristics: obesity (body mass index $>30 \mathrm{~kg} / \mathrm{m}^{2}$ ), cervical immobilisation or facial trauma.

5. Time period (before the COVID-19 pandemic vs during or after the COVID-19 pandemic).

In addition to the variables above, which can be assessed prior to enrolment, we will perform exploratory analyses examining additional potential effect modifiers that are intended to represent baseline variables, but which are collected after enrolment, and therefore have the potential to be affected by study group assignment. These include:

1. Laryngoscope type (direct laryngoscope (without video capability) vs video laryngoscope (with video capability)).

2. Presence body fluids obscuring glottic view (yes vs no).

3. Cormack-Lehane grade of view (1 vs 2-4).

\section{Sensitivity analyses of the primary outcome}

To assess the robustness of the findings, we will repeat the main analysis of the primary outcome in several alternatives to the overall intention-to-treat population. First, we will repeat the main analysis of the primary outcome among only those patients for whom a non-hyperangulated laryngoscope blade was used on the first attempt at intubation. Second, operators may choose to deviate from the assigned device for the safety of the patient after obtaining a laryngeal view. To address this, we will repeat the main analysis of the primary outcome for all patients, but will assign failure to the first intubation attempt for patients in whom the operator crossed over from the assigned device to the non-assigned device. Third, we will repeat the main analysis of the primary outcome, including only cases in which primary outcome data from the independent observer is complete (ie, excluding cases in which the operator's self-report of whether there was successful intubation on the first attempt defined the primary outcome for that patient). Fourth, because prior intubating experience may influence success with both devices, we will repeat the main analysis of the primary outcome, excluding cases where the operator had $\leq 10$ total prior intubations. Fifth, because prior experience with using a bougie may influence successful intubation in the bougie group, we will repeat the main analysis of the primary outcome, excluding cases where the operator had $\leq 5$ prior intubations while using a bougie. Sixth, we will perform a sensitivity analysis that defines successful intubation on the first attempt as successful tracheal intubation during the first insertion of the laryngoscope blade, regardless of the number of insertions of a bougie or endotracheal tube.

\section{Analysis of the secondary outcome}

For the secondary outcome, severe hypoxaemia (lowest oxygen saturation $<80 \%$ ), we will perform an unadjusted, intention-to-treat comparison of patients randomised to the bougie group versus patients randomised to the endotracheal tube with stylet group, using a $\chi^{2}$ test.

\section{Analyses of exploratory outcomes}

For all prespecified exploratory outcomes, we will conduct unadjusted, intention-to-treat analyses comparing patients randomised to the bougie to patients randomised to the endotracheal tube with stylet. Continuous outcomes will be compared with the Wilcoxon rank sum test and categorical variables with a $\chi^{2}$ test. Between-group differences in continuous and categorical variables and the associated $95 \%$ CIs will be presented.

\section{Handling of missing data}

We anticipate that no data on the primary outcome will be missing. When data are missing for the secondary or exploratory outcomes, we will perform complete-case analysis, excluding cases where the data for the analysed outcome are missing. There will be no imputation of missing data for these outcomes. In adjusted analyses, missing data for covariates will be imputed using multiple imputations.

\section{Trial status}

The BOUGIE trial is a pragmatic, prospective, multicentre, non-blinded randomised clinical trial comparing use of a bougie to use of an endotracheal tube with stylet for tracheal intubation of critically ill adults in the ED and ICU. Patient enrolment began on 29 April 2019.

\section{Pause in enrolment}

Over the first 10 months of enrolment, four patients were enrolled and subsequently found to be prisoners. On 28 February 2020, we paused enrolment to evaluate and improve enrolment procedures with a goal of preventing the enrolment of ineligible patients. The decision was made to extend the pause in enrolment during the early stages of the COVID-19 pandemic when enrolment was felt to be infeasible. Enrolment was resumed on 24 August 2020 with introduction of a new preprocedural 'time out' which requires the verbal recitation of eligibility criteria prior to enrolment to prevent subsequent enrolments of ineligible patients.

\section{Ethics and dissemination}

Waiver of informed consent

Critically ill patients undergoing tracheal intubation in the ED or ICU are at significant risk for morbidity and mortality from their underlying illness. Most patients undergoing tracheal intubation in routine clinical care receive intubation using either a bougie or an endotracheal tube with stylet on the first attempt. Any benefits or risks of these two approaches are experienced by patients undergoing tracheal intubation in clinical care, outside the context of research. As a requirement for enrolment in the BOUGIE trial, the patient's treating clinician must believe that either a bougie or an endotracheal tube with stylet would be a safe and reasonable approach for the patient (otherwise the patient is excluded). Therefore, making the decision between the two approaches randomly (by study group assignment) rather than by a provider who thinks either approach is safe and 
reasonable for the patient was expected to pose no more than minimal additional risk.

The investigators also determined that obtaining informed consent for participation in the study would be impracticable. Tracheal intubation of acutely ill patients is a time-sensitive procedure. Despite the availability of an informed consent document for the intubation procedure in clinical care, the risks and benefits of the procedure are infrequently discussed and the informed consent document for the procedure in clinical care is infrequently completed before the procedure due to its time-sensitive nature, the impairments induced by the patients' critical illness and the frequent absence of surrogate decision-makers.

Because the study was expected to pose minimal risk and prospective informed consent was considered to be impracticable, a waiver of informed consent was requested and granted from the Single Institutional Review Board at Vanderbilt University Medical Center (reference number 182123). This is consistent with previous randomised trials comparing alternative approaches to tracheal intubation commonly used in clinical care. ${ }^{71016-21}$

\section{Information for patients and families}

Information regarding the study is made available to patients and families by at least one of the following mechanisms, with the choice between the mechanisms determined by the local context assessment of the site institutional review board and site principal investigators: (1) a patient and family notification sheet provided to each patient and family following enrolment, informing the patient of their enrolment and describing the study; (2) a patient and family information sheet posted in at least three publicly visible locations within the study unit containing general information about the study and contact information for the research team for additional questions or concerns or (3) a patient and family information sheet provided to each patient and family on admission as part of an 'admission packet' containing general study information and contact information for the research team for additional questions or concerns.

\section{Protocol changes}

Any further amendments to the protocol will be recorded on ClinicalTrials.Gov as per SPIRIT guidelines. See online supplemental file 1 , section 8 , for more details on how protocol changes will be handled.

\section{Dissemination plan}

Trial results will be submitted to a peer-reviewed journal and will be presented at one or more scientific conferences.

\section{Author affiliations}

${ }^{1}$ Department of Emergency Medicine, Hennepin County Medical Center, Minneapolis, Minnesota, USA

${ }^{2}$ Department of Medicine, Division of Allergy, Pulmonary and Critical Care Medicine, Vanderbilt University School of Medicine, Nashville, Tennessee, USA
${ }^{3}$ Department of Emergency Medicine, Vanderbilt University Medical Center, Nashville, Tennessee, USA

${ }^{4}$ Department of Emergency Medicine, University of Colorado Denver School of Medicine, Aurora, Colorado, USA

${ }^{5}$ Department of Medicine, Division of Allergy, Pulmonary and Critical Care Medicine, The University of Alabama at Birmingham, Birmingham, Alabama, USA ${ }^{6}$ Pulmonary Section, Birmingham Veteran's Affairs Medical Center, Birmingham, Alabama, USA

${ }^{7}$ Department of Emergency Medicine, Denver Health Medical Center, Denver, Colorado, USA

${ }^{8}$ Department of Emergency Medicine, Wake Forest Baptist Medical Center, WinstonSalem, North Carolina, USA

${ }^{9}$ Department of Emergency Medicine, The University of Alabama at Birmingham, Birmingham, Alabama, USA

${ }^{10}$ Department of Medicine, Division of Pulmonary, Allergy \& Critical Care Medicine, The University of Alabama at Birmingham, Birmingham, Alabama, USA

${ }^{11}$ Department of Pulmonary/Critical Care Medicine, Ochsner Health System, New Orleans, Louisiana, USA

${ }^{12}$ Department of Anesthesiology and Pain Medicine, University of Washington, Seattle, Washington, USA

${ }^{13}$ Department of Emergency Medicine, Lincoln Medical Center, Bronx, New York, USA

${ }^{14}$ Department of Anesthesiology, Vanderbilt University Medical Center, Nashville, Tennessee, USA

${ }^{15}$ Department of Emergency Medicine, University of Washington, Seattle, Washington, USA

${ }^{16}$ Department of Internal Medicine, University of lowa Hospitals and Clinics Pathology, lowa City, lowa, USA

${ }^{17}$ Department of Medicine, Section of Pulmonary, Critical Care, Allergy and Immunologic Disease, Wake Forest School of Medicine, Winston-Salem, North Carolina, USA

${ }^{18}$ Department of Biostatistics, Vanderbilt University Medical Center, Nashville, Tennessee, USA

${ }^{19}$ Section of Pulmonary/Critical Care Medicine \& Allergy/Immunology, Louisiana State University, New Orleans, Louisiana, USA

${ }^{20}$ Department of Medicine, Division of Pulmonary/Critical Care Medicine, Hennepin County Medical Center, Minneapolis, Minnesota, USA

Twitter Sheetal Gandotra @SGandotraMD, Stacy A Trent @drstrent and Andrew Latimer @alatimer13

Collaborators Non-byline authors: Christopher Barnes, MD (University of Washington Harborview Medical Center); Alicia K. Cupelo, MSW (Denver Health Medical Center); Paige DeVries, BS (Hennepin County Medical Center); Sally Dye, RN (Vanderbilt University Medical Center); Andrea Fletcher, RN (Vanderbilt University Medical Center); Christopher S. Gray, RN (Vanderbilt University Medical Center); Kevin High, RN, MPH (Vanderbilt University Medical Center); Michael C. Kurz, MD, MS (University of Alabama at Birmingham Medical Center); Carol L. Lyle, MPH, PA-C (Denver Health Medical Center); Robert Mitchell, RRT (University of Colorado School of Medicine); Justin Oeth, RN, MSN (Vanderbilt University Medical Center); Erika L.W. Rice, D0 (Wake Forest School of Medicine); Sarah W. Robison, MD (University of Alabama at Birmingham Medical Center); Alexandra Schick, MD (Louisiana State University School of Medicine); Jamie Stang, BS (Hennepin County Medical Center); Andrew M. Walters, MD (University of Washington Harborview Medical Center); Nathaniel D. Westphal, MD (Wake Forest School of Medicine). Collaborators: Tak Watase, MD MBA (University of Washington Harborview Medical Center); Bradley Lloyd, RRT-ACCS (Vanderbilt University Medical Center); Vijay Krishnamoorthy, MD, PhD (Duke University School of Medicine); Raquel R Bartz, MD, MMCi (Duke University School of Medicine); William C Fox, MD (Duke University School of Medicine); John Whittle, MBBS, MD, FHEA, FRCA, FFICM (Duke University School of Medicine); Bret D. Alvis, MD (Vanderbilt University Medical Center); Stephanie C. Demasi, MD (University of Alabama at Birmingham Medical Center); Laura E. Goyack, MD (University of Alabama at Birmingham Medical Center); Kristy K. Ford, MD (Wake Forest School of Medicine); Trevor S. Mattox, MD (Wake Forest School of Medicine); Anna Altz-Stamm RN, BSN, CCRN (University of Alabama at Birmingham Medical Center); Cristina Bardita, MD, PhD (University of Alabama at Birmingham Medical Center); Mary Clay Boone RN, BSN (University of Alabama at Birmingham Medical Center); Joe W. Chiles III, MD (University of Alabama at Birmingham Medical Center); Kristina Collins RN, BSN (University of Alabama at Birmingham Medical Center); Abby Drescher RN, BSN (University of Alabama at Birmingham Medical Center); Kevin G. Dsouza, MD (University of Alabama at 
Birmingham Medical Center); Janna Dunn, RN, ADN (University of Alabama at Birmingham Medical Center); Stacy Ejem, MD (University of Alabama at Birmingham Medical Center); Josh Gautney, MD (University of Alabama at Birmingham Medical Center); Nicole Harris, RN, ADN (University of Alabama at Birmingham Medical Center); Savannah Herder, RN, BSN (University of Alabama at Birmingham Medical Center); Tamer Hudali, MD, MPH (University of Alabama at Birmingham Medical Center); R. Chad Wade, MD (University of Alabama at Birmingham Medical Center); Rutwij Joshi, MBBS (University of Alabama at Birmingham Medical Center); Daniel Kelmenson, MD (University of Alabama at Birmingham Medical Center); Anne Merrill Mason RN, BSN (University of Alabama at Birmingham Medical Center); Scott R. Merriman, MD (University of Alabama at Birmingham Medical Center); Takudzwa Mkorombindo, MD (University of Alabama at Birmingham Medical Center); Megan Moore, RN, MSN (University of Alabama at Birmingham Medical Center); Jada Nowak, RN, BSN (University of Alabama at Birmingham Medical Center); Kate O'Connor, DO (University of Alabama at Birmingham Medical Center); Sheylan D. Patel, MD (University of Alabama at Birmingham Medical Center); G. Bruno Pereira, MD, PhD (University of Alabama at Birmingham Medical Center); Lisa Sarratt RN, BSN (University of Alabama at Birmingham Medical Center); Tabitha Stewart RN, BSN (University of Alabama at Birmingham Medical Center); William S. Stigler, MD (University of Alabama at Birmingham Medical Center); Kadambari Vijaykumar, MBBS (University of Alabama at Birmingham Medical Center); Gina White RN, BSN (University of Colorado School of Medicine); Anthony Defebio (University of Colorado School of Medicine); Jennifer Friedel (University of Colorado School of Medicine); Feysel Mohamed (University of Colorado School of Medicine); Karina Nava (University of Colorado School of Medicine); Angela Otoo (University of Colorado School of Medicine); Christian Perez (University of Colorado School of Medicine) and Cori Withers (University of Colorado School of Medicine).

Contributors Approved the final version of this manuscript and critical revision of the manuscript for important intellectual content: all authors. Study concept and design: BD, MWS, WHS, DJ, TWR, MEP and JDC. Acquisition of data: MWS, WHS, AAG, S Gandrota, SAT, LMS, JPG, DBP, MRW, DJV, AMJ, JRW, CH, JSL, MPH, DWR, S Gulati, IB, DJ, TWR and JDC. Drafting of the manuscript and study supervision: BD, MWS, MEP and JDC

Funding JDC was supported in part by the National Heart, Lung, and Blood Institute (NHLBI) (K12HL133117 and K23HL153584). DWR was supported in part by the University of Alabama at Birmingham Walter Frommeyer Jr. Fellowship in Investigative Medicine and by the Department of Veteran's Affairs (VISN 7 Research Development Award). MWS was supported in part by the NHLBI (K23HL143053). Data collection used the Research Electronic Data Capture tool developed and maintained with Vanderbilt Institute for Clinical and Translational Research grant support (UL1 TR000445 from NCATS/NIH). The funding institutions had no role in (1) conception, design or conduct of the study, (2) collection, management, analysis, interpretation or presentation of the data or (3) preparation, review or approval of the manuscript.

Competing interests None declared.

Patient consent for publication Not required.

Provenance and peer review Not commissioned; externally peer reviewed.

Supplemental material This content has been supplied by the author(s). It has not been vetted by BMJ Publishing Group Limited (BMJ) and may not have been peer-reviewed. Any opinions or recommendations discussed are solely those of the author(s) and are not endorsed by BMJ. BMJ disclaims all liability and responsibility arising from any reliance placed on the content. Where the content includes any translated material, BMJ does not warrant the accuracy and reliability of the translations (including but not limited to local regulations, clinical guidelines, terminology, drug names and drug dosages), and is not responsible for any error and/or omissions arising from translation and adaptation or otherwise.

Open access This is an open access article distributed in accordance with the Creative Commons Attribution Non Commercial (CC BY-NC 4.0) license, which permits others to distribute, remix, adapt, build upon this work non-commercially, and license their derivative works on different terms, provided the original work is properly cited, appropriate credit is given, any changes made indicated, and the use is non-commercial. See: http://creativecommons.org/licenses/by-nc/4.0/.

\section{ORCID iDs}

Brian Driver http://orcid.org/0000-0002-7141-0256
Matthew W Semler http://orcid.org/0000-0002-7664-8263

Sheetal Gandotra http://orcid.org/0000-0002-5475-9704

Jason R West http://orcid.org/0000-0002-0060-0861

Derek W Russell http://orcid.org/0000-0002-2716-1344

\section{REFERENCES}

1 Sakles JC, Chiu S, Mosier J, et al. The importance of first pass success when performing orotracheal intubation in the emergency department. Acad Emerg Med 2013;20:71-8.

2 Hasegawa K, Shigemitsu K, Hagiwara Y, et al. Association between repeated intubation attempts and adverse events in emergency departments: an analysis of a multicenter prospective observational study. Ann Emerg Med 2012;60:749-54.

3 Mort TC. Emergency tracheal intubation: complications associated with repeated laryngoscopic attempts. Anesth Analg 2004;99:607-13.

4 Duggan LV, K.S. M, Griesdale DE, et al. Complications increase with greater than one endotracheal intubation attempt: experience in a Canadian adult tertiary-care teaching center. J Clin Anesth 2014;26:167.

5 Brown CA, Bair AE, Pallin DJ, et al. Techniques, success, and adverse events of emergency department adult Intubations. Ann Emerg Med 2015;65:363-70.

6 Park L, Zeng I, Brainard A. Systematic review and meta-analysis of first-pass success rates in emergency department intubation: creating a benchmark for emergency airway care. Emerg Med Australas 2017;29:40-7.

7 Janz DR, Semler MW, Lentz RJ, et al. Randomized trial of video laryngoscopy for endotracheal intubation of critically ill adults. Crit Care Med 2016;44:1980-7.

8 Driver B, Dodd K, Klein LR, et al. The Bougie and First-Pass Success in the Emergency Department. Ann Emerg Med 2017;70:473-8.

9 Alkhouri $\mathrm{H}$, Vassiliadis J, Murray M, et al. Emergency airway management in Australian and New Zealand emergency departments: a multicentre descriptive study of 3710 emergency Intubations. Emerg Med Australas 2017;29:499-508.

10 Driver BE, Prekker ME, Klein LR, et al. Effect of use of a Bougie vs endotracheal tube and Stylet on First-Attempt intubation success among patients with difficult airways undergoing emergency intubation: a randomized clinical trial. JAMA 2018;319:2179-89.

11 Chan A-W, Tetzlaff JM, Gøtzsche PC, et al. Spirit 2013 explanation and elaboration: guidance for protocols of clinical trials. BMJ 2013;346:e7586.

12 Levitan RM. Avoiding common laryngoscopy errors, part II, 2020. Available: http://epmonthly.com/article/avoiding-commonlaryngoscopy-errors-part-ii/

13 Cormack RS, Lehane J. Difficult tracheal intubation in obstetrics. Anaesthesia 1984;39:1105-11.

14 Driver BE, Prekker ME, Moore JC, et al. Direct versus video laryngoscopy using the C-MAC for tracheal intubation in the emergency department, a randomized controlled trial. Acad Emerg Med 2016;23:433-9.

15 RStudio Team. RStudio: integrated development for R. Boston, MA: RStudio, Inc, 2015. http://www.rstudio.com/

16 Tekwani KL, Watts HF, Sweis RT, et al. A comparison of the effects of etomidate and midazolam on hospital length of stay in patients with suspected sepsis: a prospective, randomized study. Ann Emerg Med 2010;56:481-9.

17 Jabre P, Combes X, Lapostolle F, et al. Etomidate versus ketamine for rapid sequence intubation in acutely ill patients: a multicentre randomised controlled trial. Lancet 2009;374:293-300.

18 Semler MW, Janz DR, Lentz RJ, et al. Randomized trial of apneic oxygenation during endotracheal intubation of the critically ill. Am J Respir Crit Care Med 2016;193:273-80.

19 Janz DR, Semler MW, Joffe AM, et al. A multicenter randomized trial of a checklist for endotracheal intubation of critically ill adults. Chest 2018;153:816-24.

20 Semler MW, Janz DR, Russell DW, et al. A multicenter, randomized trial of ramped position vs sniffing position during endotracheal intubation of critically III adults. Chest 2017;152:712-22.

21 Caputo N, Azan B, Domingues R, et al. Emergency department use of apneic oxygenation versus usual care during rapid sequence intubation: a randomized controlled trial (the ENDAO trial). Acad Emerg Med 2017;24:1387-94. 